\title{
Inovação em Turismo e Competitividade Regional: abordagem conceitual e ensaio de aplicação
}

Rosana Mara Mazaroa

\begin{abstract}
Resumo
Embora o número de estudos sobre competitividade e inovação regional tenha crescido consideravelmente na literatura, ainda persiste uma notável ausência de pesquisas que abordem a convergência entre os dois temas, especialmente no que trata da simetria entre os atributos, dimensões e escala de aplicação de cada constructo. Tendo como premissa que a inovação é um fator de competitividade para os destinos e regiões turísticas e que, portanto, são assuntos que devem ser tratados concomitantemente, este estudo aborda essa lacuna e sugere um constructo único que congregue fatores que condicionem a capacidade de inovação e a competitividade dessas regiões. A análise qualitativa do conteúdo dos diferentes modelos de competitividade de destinos em comparação com os modelos de inovação gerou resultados significativos, dentre esses a identificação de importante convergência entre fatores que condicionam ambos os fenômenos, a classificação dos fatores entre convergentes e complementares, e a proposição de um modelo holístico de inovação para análise em escala regional com recorte específico para o turismo.
\end{abstract}

Palavras-chave: Turismo; Inovação; Sistemas Regionais; Competitividade.

\begin{abstract}
Innovation in regional tourism and competitiveness: conceptual approach and application trial

Although the number of studies on regional competitiveness and innovation has considerably grown in literature, there is still a notable lack of research addressing the convergence between the two themes, especially concerning the symmetry between the attributes, dimensions, and scale of application of each construct. Taking as premise that innovation is a factor of competitiveness for tourist destinations and regions and that, therefore, they are matters that should be addressed at the same time, this study addresses this gap and suggests a single construct integrating factors that affect the capacity for innovation and competitiveness of these regions. Qualitative analysis of the content of the different models of competitiveness of destinations in comparison with the models of innovation generated significant results, among them the identification of important convergence between factors that influence both phenomena, the classification of the factors between convergent and complementary, and the proposition of a holistic model of innovation for regional-scale analysis with specific focus on tourism.
\end{abstract}

Keywords: Tourism; Innovation; Regional Systems; Competitiveness.

a. Doutora em Administração e Turismo. Mestre em Administração Pública. Graduada em Administração. Docente permanente do Programa de Pós-Graduação em Turismo da Universidade Federal do Rio Grande do Norte. Líder do Grupo de Pesquisa ESCRITUR. Natal, Rio Grande do Norte, Brasil. E-mail: rosanamazaro@uol.com.br 


\section{Resumen \\ Innovación en turismo y competitividad regional: enfoque conceptual y ensayo de aplicación}

Aunque los estudios sobre la competitividad y la innovación en las regiones han crecido considerablemente en la literatura, todavía hay una notable ausencia de investigaciones que aborden la convergencia entre las dos cuestiones, especialmente en el tratamiento de la simetría entre los atributos, las dimensiones y el rango de aplicación de cada uno de estos constructos. Con la premisa de que la innovación es un factor de competitividad para los destinos y regiones turísticas y, por lo tanto, son cuestiones que deben abordarse al mismo tiempo, este estudio aborda este vacío en las investigaciones y sugiere una única construcción que reúne a los factores que influyen en la capacidad de innovación y la competitividad de estas regiones. El análisis cualitativo del contenido de los diferentes modelos competitivos en comparación con el análisis de los modelos de innovación generó resultados significativos, entre ellos la identificación de los factores que determinan la convergencia entre ambos fenómenos: la clasificación de los factores entre convergentes y complementares y la proposición de un modelo integral de innovación para el análisis en escala regional con un enfoque específico para el turismo.

Palabras clave: Turismo; Innovación; Sistemas Regionales de Competitividad.

\section{INTRODUÇÃO}

Na virada do século, o imperativo competitivo para todos os setores produtivos foi resumido no livro de Trout (2000), que profetizava já em seu título: diferenciar ou morrer. Hoje, após mais de uma década de transição e transformação no contexto competitivo, parece ser possível atualizar a profecia para uma nova dicotomia: inovar ou morrer.

As pesquisas sobre inovação no setor de manufatura basearam os primeiros ensaios para o desenvolvimento de estudos sobre inovação no setor de serviços, ainda em um estágio inicial de conformação de um campo referencial próprio. De acordo com Hjalager (2010), a emergente economia de serviços, impulsionada em grande parte pelo desenvolvimento do software, teve sua extraordinária expansão no final do último milênio que, por sua vez, mudou radicalmente a concepção de inovação, incorporando ao conceito atributos imateriais e elementos intangíveis em sua definição e aplicação.

Se no âmbito organizacional a inovação está geralmente relacionada a novos produtos, melhoria de produtos existentes, tecnologia e processos de gestão, no nível macroeconômico está intimamente ligada com o desenvolvimento dos locais e com o bem-estar de seu povo. A inovação é agora o eixo central de estratégia competitiva não só para negócios e organizações, mas também para países e regiões.

Essa é a perspectiva de inovação que motiva este ensaio e que tenta entender seu significado e impactos na competitividade das economias regionais, neste caso, com particular interesse para o setor turístico (WEF, 2007; RODRÍGUEZ; WILLIAMS; HALL, 2014). 0 estudo é uma análise da inovação em turismo sob uma perspectiva regional, e se apoia em fatores intrínsecos que o distingue dos demais setores produtivos e da importância da integração e interdependência da cadeia de valor que compõe a oferta global de um destino, que exige abordagens e enfoques macro de inovação. 
O esforço aqui é integrar as pesquisas sobre inovação às pesquisas sobre competitividade de destinos, considerando a direta relação de influência de um fenômeno sobre o outro. Para isso, a primeira parte aborda a evolução dos estudos sobre competitividade de destinos turísticos e compara os diferentes modelos teóricos e metodológicos para identificar e categorizar o que são aqui denominados atributos básicos de competitividade de destinos.

A segunda parte é dedicada ao estado da arte sobre inovação, especialmente na compreensão de seu significado para países e regiões e nos esforços para tangibilizar o conceito e identificar os atributos básicos de inovação em destinos. A terceira parte trata do cotejamento entre os dois constructos, comparando e categorizando os atributos de cada um, para ao final propor um modelo referencial de inovação sob a perspectiva do turismo e adequado à escala regional.

O modelo proposto pode ser aplicado como base metodológica para caracterização de sistemas regionais de inovação em turismo e para avaliação da capacidade dessas regiões para inovar. Os resultados dessa avaliação permitirão constituir tipologias de regiões de acordo com o potencial para inovar, e poderão orientar o planejamento e gestão de destinos para escolha de estratégias ajustadas a um novo e desafiador contexto competitivo.

\section{REVISÃO DA LITERATURA}

\section{Imperativos competitivos para destinos turísticos}

O avanço na compreensão dos fatores que determinam condições favoráveis de competitividade para os destinos turísticos, principalmente pela inclusão de critérios de sustentabilidade como condicionantes estratégicos, foi traduzido por meio de distintos modelos teórico-metodológicos que sintetizam o complexo sistema turístico e sua rede de relações (DWYER; KIM, 2003; GENEST; LEGG, 2003; GOOROOCHURN; SUGIYARTO, 2005; RITCHIE; CROUCH, 2003; WEF, 2007).

O Quadro 1 apresenta as principias características de cada modelo e compara seus componentes para demonstrar a convergência entre seus atributos e fatores determinantes da competitividade. 


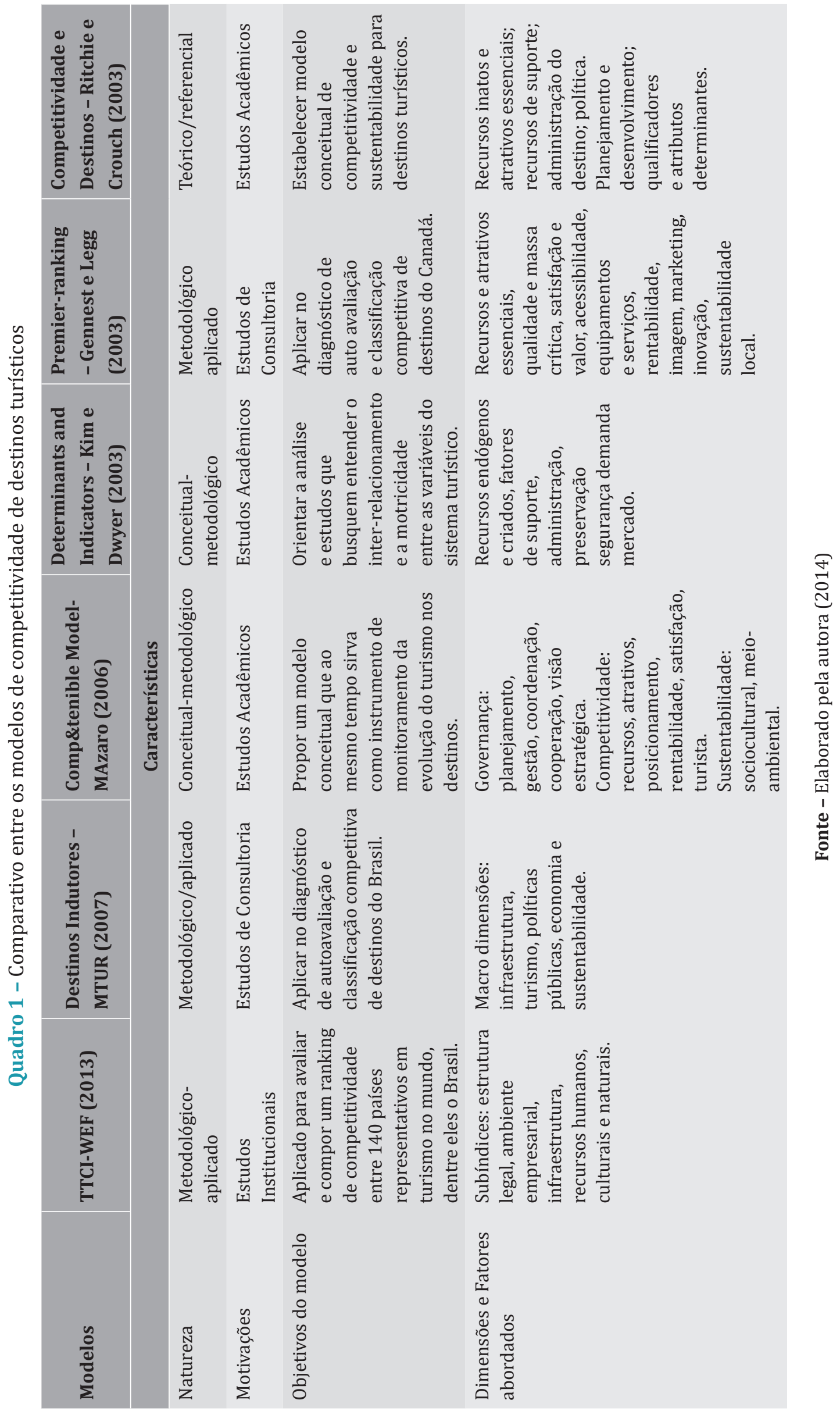


Como pode ser observado no quadro, os fatores, atributos ou variáveis que compõem esse contexto parecem já identificados e conceituados apropriadamente no modelo de Ritchie e Crouch (2003), na essência o mais teórico dentre todos aqui analisados, tendo o mérito de ser exaustivo na dimensão em que deve ser. As metodologias sugeridas pelo premier-ranking de Genest e Legg (2003), aplicadas em destinos do Canadá e utilizadas pela Fundação Getulio Vargas no Brasil (BRASIL; FGV; SEBRAE, 2008), são importantes para a definição das dimensões e atributos da competitividade para destinos, e confirmam a multidimensionalidade de fatores passíveis de ingerência e impactos para o turismo.

Porém, são modelos de avaliação dicotômicos e próprios para o exercício de um diagnóstico que não se ocupa de avaliar de maneira qualitativa ou explicativa as variáveis. Desde essas conformações, os estudos evoluíram para tentativas de compreensão das inter-relações entre as variáveis e dimensões do turismo local sob uma perspectiva competitiva e sustentável, bem como se empreenderam esforços na tentativa de avaliação e medida de padrões de competitividade relacionados a parâmetros globais ou referenciais.

Seguindo essa via de proposição, o modelo de Dwyer e Kim (2003) vai além de definir as dimensões e fatores críticos para o turismo local e sugere uma metodologia para avaliação da competitividade de destinos pela utilização de ferramentas estatísticas que conseguem identificar ou quantificar a motricidade das variáveis, ou seja, explicar sistemicamente a lógica entre decisão e ação.

Os estudos que utilizaram o modelo dos autores revelaram importante influência das variáveis de governança sobre os resultados e conquistas competitivas do destino, incluindo fatores de impacto sobre aspectos globais, relacionados ao desenvolvimento e sustentabilidade local. Esses estudos realçam o papel da gestão como a responsável pelas decisões que vão impactar o turismo nos destinos, que, por sua vez, vai influenciar o desempenho competitivo em curto prazo e, ao mesmo tempo, determinar o vetor de desenvolvimento para o futuro (DWYER; KIM, 2003).

Por sua vez, o Comp\&tenible (MAZARO, 2010) tem como princípio a motricidade das variáveis de gestão sobre a competitividade e sustentabilidade de destinos turísticos, sintetizando e sistematizando em distintas dimensões os fatores que condicionam o ajuste estratégico entre as oportunidades e determinantes macroambientais, nesse contexto, traduzidos pelos imperativos da sustentabilidade. Tais dimensões representam o que se antecipou afirmar sobre a motricidade dos componentes de gestão sobre resultados e impactos do turismo nos destinos.

Com foco específico no setor turístico, o Fórum Econômico Mundial tem promovido desde 2005 estudos sobre o desempenho competitivo em turismo de diferentes países de todas as regiões do mundo, que resultam no Travel \& Tourism Competitiviness Index (T\&TCI). O T\&TCI consiste em uma metodologia de avaliação considerada como "uma ferramenta estratégica global para medir os fatores e políticas que tornam atrativo desenvolver o setor de turismo e viagens em diferentes países." (WEF, 2011, p. xiv, tradução nossa).

O objetivo do T\&TCI é fornecer uma ferramenta estratégica abrangente para medir "o conjunto de fatores e políticas que permitem o desenvolvimento sustentável do setor de Turismo \& Viagem, que, por sua vez, contribui para o de- 
senvolvimento e competitividade de um país" (WEF, 2015, p. v, tradução nossa). Ao fornecer detalhada avaliação dos ambientes de Travel \& Tourism (T\&T) dos países em todo o mundo, os resultados podem ser usados por todas as partes interessadas em trabalhar em conjunto para melhorar a competitividade da indústria em suas economias nacionais.

\section{Abordagens de inovação em turismo}

Inovação é o principal desafio competitivo na atualidade e continuará sendo no futuro. É um fenômeno que interessa e afeta todos os segmentos da sociedade. A preocupação com a inovação ganha relevância à medida que as pressões da competitividade crescem na economia globalizada, e partes da cadeia de valor ou cadeias de valor inteiras vão sendo deslocadas para economias com condições de produtividade mais atraentes às vistas do capital. Mesmo o setor de serviços, tradicionalmente considerado não transacionável, está adaptando-se às mudanças da economia internacional e às mudanças nas atividades econômicas globalizadas, alinhando-se com uma nova ordem (OECD, 2006).

A definição proposta por Schumpeter (apud SARKAR, 2010) é ainda a mais referenciada e utilizada quando o esforço é o de compreender o que é inovação. Segundo o autor, inovação implica dois elementos fundamentais: criatividade e ideias novas. 0 termo "inovar" está intimamente ligado aos verbos "implementar", "empreender", "viabilizar", "efetivar" e "consolidar" ideias. Em poucas palavras, a inovação é uma invenção viável, realizável e rentável.

A inovação em turismo também tem ganhado importância e atualmente é tema de interesse para instituições nacionais e internacionais de pesquisa e desenvolvimento, governos, investidores e, certamente, o próprio turista (HJALAGER, 2010). Estes estudos, classificados como serviços relacionados ao deslocamento e à hospitalidade, são ainda mais recentes, e, à margem dos avanços da pesquisa acadêmica, o setor de turismo tem demonstrado ao longo do tempo uma importante capacidade de inovação e criatividade, conceitos intimamente relacionados na literatura corrente (HALL; WILLIAMS, 2008).

No decorrer das últimas duas décadas tem havido crescente foco no tema da inovação no setor turístico. De acordo com Hjalager (2010), a inovação em turismo se classifica em:

- Inovações de produtos ou serviços: se referem às mudanças observadas diretamente pelo cliente e consideradas como novas, tanto no sentido de nunca terem sido vistas antes ou novas para a empresa particular ou de destino.

- Inovações de processo: se referem normalmente às iniciativas de bastidores que visam à eficiência, produtividade e fluxo. Os investimentos em tecnologia são a âncora do processo de inovação, às vezes em combinação com layouts regenerados para operações de trabalho manual.

- Inovações gerenciais: novas formas de organizar a colaboração interna, orientando e capacitando pessoal, construindo carreiras e compensando o trabalho com remuneração e benefícios, também podendo ser destinadas 
a melhorar a satisfação no trabalho e fomentar o conhecimento interno e recursos de competência.

- Inovações de gestão: mudanças na maneira como a comunicação global da organização com os clientes é feita, e como os relacionamentos entre prestador do serviço e cliente são construídos e mantidos.

- Inovações institucionais: constituição de novas competências ou combinação daquelas já estabelecidas entre organizações para colaboração interinstitucional, efetivado por meio de acordos bilaterais para a formação de alianças e redes.

As inovações institucionais constituem o foco deste estudo, considerando que as estruturas e diretrizes de gestão de destinos são sempre orientadas para a participação e cooperação interinstitucional e têm sua essência na combinação de competências. De acordo com Lundvall et al. (2002), nessa perspectiva, a inovação é um fenômeno intrinsecamente territorial e localizado, altamente dependente de recursos que são ligados às localidades específicas e cuja reprodução seria impossível em outro lugar. 0 autor define quatro forças que impulsionam o desenvolvimento: a organização flexível da produção; a difusão das inovações e do conhecimento; a mudança e adaptação das instituições e o planejamento urbano do território.

A interação entre essas forças produziria a sinergia necessária capaz de alavancar um desenvolvimento endógeno que, por sua vez, permitiria uma nova alternativa de crescimento econômico não mais construído de fora para dentro, mas resultado de uma dinâmica econômica local. Ao mesmo tempo, esse desenvolvimento se fundamentaria na valorização das éticas e das expressões culturais locais, necessárias à consolidação de práticas cooperativas, ao crescimento da confiança entre indivíduos e grupos, além da proteção ao patrimônio cultural e ambiental dos territórios envolvidos (LONGHI, 2005).

A União Europeia estabeleceu como principal objetivo para as próximas décadas liderar, competir e prosperar como uma economia mais verde, de crescimento rápido e sustentável, estimulando a criação de elevados níveis de emprego baseados no conhecimento e progresso social. Para isso, instituiu a pesquisa e a inovação como eixo central de suas políticas de desenvolvimento ao longo desse horizonte temporal, acentuando sua importância como um dos principais desafios competitivos atuais para países e regiões. Embora crescimento, emprego e competitividade fossem considerados grandes desafios para a Europa já na década passada, a inovação somou-se aos elementos centrais da estratégia europeia para enfrentar os novos desafios competitivos colocados pelo mundo pós-crise (SOETE, 2010).

A tradição europeia no uso de modelos aplicados a macro setores, bem como modelos para medir e simular o impacto de políticas públicas de diferentes naturezas, preconizou o desenvolvimento e proposição de uma metodologia denominada Innovation Union Scoreboard (IUS), que serve de método de monitoramento do desempenho competitivo em termos de inovação nos países e de ferramenta de avaliação da implementação dos objetivos do Europe 2020 Innovation Union, plano de desenvolvimento europeu com foco na inovação (UNU-MERIT, 2015). Essencialmente, os indicadores, que serão explorados detalhadamente adiante, traduzem as condições básicas e fundamentais que permitem que os países sejam mais ou menos inovadores. 
Importante nesses constructos é o esforço para tangibilizar conceitos tão complexos e difusos, como o de inovação, e traduzir em indicadores aqui sistematizadamente dispostos na sequência de um processo sistêmico, dinâmico e inter-relacionado. Esses elementos combinados em um espaço geográfico determinado podem constituir um sistema regional favorável à inovação. De acordo com Hall e Williams (2008), a partir de uma perspectiva de inovação regional, o turismo no contexto dessa estratégia é um facilitador da mobilidade humana, estimula a formação de redes e pode contribuir efetivamente para a inovação regional e desenvolvimento em longo prazo, através de estabelecimento de ligações com outros setores e vetores do desenvolvimento territorial.

Hall e Williams (2008) sugerem uma tipologia de sistemas regionais de inovação (RIS, na sigla em inglês), estabelecida a partir de uma avaliação das trajetórias tecnológicas relativas das regiões e de sua diversidade. A capacidade de aproveitar competências e gerar inovação é caracterizada conforme segue:

- Genuínas: Essas são regiões onde novas combinações realmente têm lugar e as melhores práticas ocorrem. Todas as etapas dos ciclos de inovação podem existir dentro delas, assim como seus principais atores são capazes tanto de incorporar novidades como de inspirar outras inovações, criando assim um ciclo complementar virtuoso. Essas regiões também mantêm relações competitivas e colaborativas com outras regiões líderes em seus segmentos.

- Incrementais: Essa categoria de SRI tem como principal característica a capacidade de adotar inovações externas ainda no início do ciclo de vida e aperfeiçoá-las ou incrementá-las. Com isso, desenvolvem estratégias competitivas diferenciais, o que potencialmente as leva a melhor exploração de suas competências regionais e incremento na qualidade e oferta de produtos e serviços.

- Seguidoras: Essas são as SRI em que as inovações se difundem relativamente devagar. Adotam geralmente estratégias de imitação que produzem produtos desenvolvidos sem significativas melhorias na inovação original, mas que ainda tem um mercado. No caso de serviços como o turismo, uma abordagem real da reprodução pode ser inovadora em mercados regionais, mas não pode sustentar vantagem competitiva em maior escala geográfica e de valor.

Quanto à estratégia competitiva, regiões genuinamente inovadoras adotam estratégias high-road, fundamentalmente baseadas na aprendizagem, conectividade, comunicação e outros fatores intangíveis, tais como capital intelectual e capacidade institucional, que refletem em altos níveis de comodidade para visitantes e moradores, e na valorização da diversidade (HALL; WILLIAMS, 2008). No outro polo, as regiões seguidoras adotam estratégias low-road, que tem foco em fatores tradicionais e de vantagem comparativa (RITCHIE; CROUCH, 2003), como a localização, terra, trabalho, capital, infraestrutura e acesso a mercados e outros elementos-chave da produção.

Estratégias de low road são geralmente consideradas como atreladas a uma visão de crescimento de propriedade preocupadas com a "embalagem" do produto local, e pela geração de factoides para garantir a atenção da mídia. Para os destinos turísticos, esse tipo de estratégia pode levar à réplica de uma série de características do local que provocam a homogeneidade do mercado e oferta indiferenciada. Repre- 
sentam, ainda, o reflexo das escolhas dos gestores que utilizam os chamados "off-the-shelf" (sem práticas próprias) ou "best practice" (melhores práticas de outros).

Esse processo foi descrito por Doel_e Hubbard_(2002) como sendo parte do chamado "empreendedorismo urbano", em que muitas das inovações e investimentos destinados a fazer determinados locais mais atraentes para moradores e turistas são rapidamente imitados em outros lugares, tornando assim efêmera a vantagem competitiva dentro de uma categoria de cidades e destinos. Isso implica dizer que a competitividade regional e as soluções desenhadas para uma determinada localidade, quando inspiradas a partir da experiência de outras regiões, podem pecar pela falta de originalidade e de exploração de competências regionais genuínas.

Importante nessa classificação são as características gerais que configuram e podem diferenciar uma região de outra, reforçando os componentes intangíveis relacionados ao capital social e ao paradigma de desenvolvimento como principais indutores e sustentadores de uma situação mais favorável a iniciativas inovadoras e à criação de diferenciais competitivos inimitáveis. Desse modo, os níveis regionais e locais são entendidos aqui como importantes espaços para a inovação e isso é especialmente relevante para o turismo, uma vez que a sua oferta está baseada nos atributos da localidade e cujo movimento estratégico reforça a originalidade como um determinante competitivo capaz de sustentar diferenciais.

\section{METODOLOGIA}

Caracteriza-se como um estudo teórico-empírico de cunho qualitativo. Trata-se de pesquisa conceitual (XIN; TRIBE; CHAMBERS, 2013) que utiliza método comparativo entre constructos teóricos já consolidados para sugerir tipologia conceitual de sistema regional de inovação com recorte especifico para o turismo, aqui denominada Ristur. Utilizando a análise de conteúdo de fontes como livros, artigos científicos, ensaios acadêmicos, estudos de caso e demais publicações indexadas e qualificadas sobre competitividade e inovação em turismo, foi possível identificar diferentes constructos e categorizá-los segundo critérios de abrangência, foco e objetivos.

Esta análise exploratória serviu para a primeira categorização dos modelos e facilitou a comparação entre dimensões, subdimensões e variáveis específicas utilizadas para explicar cada fenômeno separadamente. A comparação foi usada para a escolha daqueles modelos considerados de maior abrangência, importância e representatividade, ao reunir os atributos de competitividade, de um lado, e inovação, de outro. Foram escolhidos para análise em profundidade e cotejamento entre conteúdos o IUS (UNO-MERIT, 2015) e o T\&TCI (WEF, 2015).

0 confronto entre modelos e sua análise foram realizados a partir de seus principais pilares, por considerar que representam um conjunto de indicadores relacionados entre si e que são capazes de refletir uma determinada condição ou situação de forma mais abrangente do que a comparação entre indicadores específicos. Por estar em uma dimensão intermediária do modelo, considera-se que atende ao critério de abrangência, uma vez que não é tão global quanto um subindex e nem tão específica quanto um indicador.

As dimensões foram analisadas individualmente e depois comparadas entre si, a fim de identificar similaridades, complementaridades ou discrepâncias. Essa análise do conteúdo em profundidade permitiu uma primeira classificação dos 
atributos em dois grupos distintos: os convergentes e os complementares. Uma segunda classificação foi então sugerida a partir da agregação de atributos em três subdimensões de análise, a exemplo dos modelos analisados, reunindo o que se acredita serem os condicionantes para a inovação em regiões turísticas e que compõem o modelo referencial aqui proposto.

\section{RESULTADOS E DISCUSSÃO}

A inovação é o ponto focal das políticas atuais por conta de sua notória contribuição para a competitividade (RODRÍGUEZ; WILLIAMS; HALL, 2014). Portanto, como indicado antes, a intenção aqui é discutir esses dois temas como integrados e convergentes, especialmente no contexto dos destinos turísticos. A partir do confronto entre os modelos representativos de ambos os constructos foi possível realizar a primeira classificação dos atributos em convergentes e complementares. O Quadro 2 traz as macro dimensões e componentes de cada modelo, sintetizando a comparação.

Quadro 2 - Atributos de competitividade do T\&TCI e IUS

\begin{tabular}{|l|l|}
\hline Ambiente favorável & Habilitadores \\
\hline $\begin{array}{l}\text { 1. Ambiente de negócios (12) } \\
\text { 2. Segurança e da segurança (5) }\end{array}$ & 1. Recursos humanos (3) \\
\hline $\begin{array}{l}\text { 3. Saúde e higiene (6) } \\
\text { 4. Recursos humanos/mercado de } \\
\text { trabalho (9) }\end{array}$ & 3. Financiamento e suporte à pesquisa (2) \\
\hline 5. Preparação de TIC (8) & 4. Investimentos em P\&D (2) \\
\hline Política de T\&T & 5. Empreendedorismo e cooperação (3) \\
\hline $\begin{array}{l}\text { 6. Priorização de viagens e turismo (6) } \\
\text { 7. Abertura international (3) }\end{array}$ & 6. Capital intelectual (4) \\
\hline 8. Competitividade em preço (4) & Resultados \\
\hline 9. Sustentabilidade ambiental (10) & 7. Inovadores (3) \\
\hline Infraestrutura & 8. Impactos econômicos (5) \\
\hline 10. Infraestrutura de transporte aéreo (6) & \\
\hline $\begin{array}{l}\text { 11. Infraestrutura portuária (7) } \\
\text { 12. Serviços turísticos (4) }\end{array}$ & \\
\hline Recursos naturais e culturais & \\
\hline 13. Recursos naturais (5) & \\
\hline 14. Recursos culturais (5) & \\
\hline
\end{tabular}

O IUS-EU foi apresentado durante o Painel Europeu da Inovação, em 2001, como uma metodologia de avaliação do desempenho em inovação dos países europeus. 0 resultado global do ranking possibilita a avaliação comparativa do desempenho da inovação dos 27 estados membros da União Europeia e de seus competidores de ponta, como Japão e Estados Unidos, por um lado, e dos emergentes pertencentes ao Brics (Brasil, Rússia, Índia, China e África do Sul) (UNU- 
-MERIT, 2011). A 14aㅡ edição, publicada em 2015, segue a mesma metodologia da primeira edição, demonstrando estabilidade e apropriação de seu conteúdo.

O IUS é medido usando um indicador composto denominado Índice de Inovação, sintetizado em três subíndices: habilitadores, atividades empresariais e resultados - oito dimensões e um total de 25 indicadores. Os números entre parênteses representam os indicadores usados para medir cada variável. 0 subíndice "habilitadores" é formado por um conjunto de indicadores que capturam os principais motores do desempenho da inovação externos às empresas, e os diferencia em três dimensões de inovação:

- Recursos humanos: inclui indicadores que medem a disponibilidade da força de trabalho altamente qualificada e instruída, como formação em nível de doutorado e publicações especializadas de alto impacto;

- Sistemas de pesquisa: mede a competitividade internacional de bases científicas, incidindo sobre publicações internacionais, citação das publicações e intercâmbio entre doutorandos;

- Fomento: mede a disponibilidade de financiamento para projetos de inovação e de investimentos, como capital de risco, bem como o apoio de governos em gastos de P\&D.

O subíndice "atividades empresariais" captura os esforços de inovação internos à empresa e avalia seu investimento em $\mathrm{P} \& \mathrm{D}$ e outros investimentos, de modo a gerar inovações. Os indicadores também captam os esforços de colaboração entre empresas inovadoras e colaboração de pesquisa entre o setor público e privado, além de diferentes formas de Direitos de Propriedade Intelectual (DPI) gerados como rendimento no processo de inovação, incluindo pedidos de patentes PCT, marcas comunitárias e desenhos ou modelos comunitários. Tendo como premissa que a inovação se configura em algo tangível, viável e rentável, a segunda dimensão de análise trata dos negócios gerados pela inovação, como consequência da combinação positiva dos fatores condicionantes, ou inputs do processo.

O terceiro subíndice se ocupa dos outputs e tenta captar os efeitos das atividades de inovação por meio de indicadores que medem o volume de introdução de inovações no mercado, abrangendo tanto as inovações tecnológicas como as não tecnológicas. 0 sucesso econômico da inovação no emprego em atividades intensivas em conhecimento e a internacionalização da inovação são também fatores considerados. Nessa perspectiva, a terceira e última dimensão do modelo completa o ciclo proposto pelo Índice, uma vez que avalia os resultados do processo com a efetividade da inovação.

Com foco específico no turismo, o T\&TCI coordenado pelo Word Economic Forum (WEF) compara a competitividade em turismo entre os países e inclui perfis detalhados para cada uma das economias estudadas. É composto por quatro subíndices, catorze pilares e noventa indicadores. Diferente do IUS, a composição do Índice sofreu algumas alterações ao longo dos anos, resultando no abandono, substituição ou incorporação de novos indicadores. Inicialmente continha três subíndices e treze pilares. A última versão, publicada em 2015, contém quatro subíndices e, desde 2011, distribui noventa indicadores em catorze pilares. 
O subíndice "ambiente favorável" captura as configurações gerais necessárias para operar em um país; o subíndice "política de T\&T" capta condicionantes relativos às políticas específicas ou aspectos estratégicos que afetam o T\&T diretamente; o subíndice "infraestrutura" avalia a disponibilidade e qualidade da infraestrutura física de cada país; e o subíndice "recursos naturais e culturais" capta os principais potenciais motivadores de viagem para o país analisado. Compilado pela primeira vez em 2007, a sua sexta edição permite que os países acompanhem a evolução das condições de competitividade ao longo do tempo e o usem como base de informação para a tomada de decisão nos destinos.

No que se refere ao conteúdo, a proposição do T\&TCI não sugere exatamente um processo sistêmico, como o faz o IUS, não sugerindo, portanto, uma relação causa-efeito entre as categorias ou subíndices de indicadores. No entanto, ainda que espontaneamente colocado, o primeiro subíndice reúne indicadores que condicionam o desenvolvimento do turismo, e que no modelo são denominados habilitadores (enablers), ou seja, representam condições básicas elementares para que o processo de desenvolvimento turístico possa ser iniciado ou alavancado.

Por sua vez e de forma intencional, o IUS também caracteriza o conjunto de indicadores que compõem o primeiro subíndice como habilitadores da inovação, o que significa que sem a presença desses condicionantes o processo de inovação pode ser seriamente comprometido, ou mesmo nulo. Essa é a primeira importante convergência entre os indicadores dos modelos, o que reforça nossa premissa de que são fenômenos inter-relacionados e interdependentes. Ainda que o IUS trate mais de indicadores relacionados à capacidade intelectual e inteligência humana e o T\&TCI incorpore também elementos estruturais, ambos os pilares ou dimensões de cada modelo se ocupam de variáveis externas ao ambiente empresarial, ou seja, com o macroambiente socioeconômico cultural.

É justamente no macroambiente onde acontecem os movimentos estratégicos importantes e que têm impacto direto sobre os fenômenos da inovação e competitividade (KIM; MAUBORGNE, 2003). 0 fator humano, devido à capacidade exclusiva de acumular capital intelectual, aparece nessa dimensão como central para a capacidade de inovar e estratégico para a competitividade das nações. As duas categorias seguintes de análise do IUS abordam indicadores relacionados diretamente com atividades empresariais e investimentos em $\mathrm{P} \& \mathrm{D}$, confirmando o princípio da efetividade inerente ao conceito de inovação. 0 T\&TCI também contempla, entre seus pilares, indicadores relacionados às atividades empresariais que compõem o setor turístico, ainda que, como já colocado, não esteja agrupado em um mesmo pilar e não siga um processo sistêmico como faz o IUS.

O IUS classifica os países estudados em quatro diferentes categorias em relação à inovação: líderes, seguidores, moderados e modestos. Isso significa que nem todos são iguais perante a classificação. A exemplo do que sugere a abordagem dos sistemas regionais de inovação, a Comissão Europeia, por meio do IUS, põe em relevo que nem todas as regiões ou países apresentam as mesmas condições para inovar e aproveitar suas competências para transformar atributos endógenos em vetores de competitividade. Desse modo, este 
trabalho se propõe a seguir uma forma de conhecer e avaliar as condições regionais que habilitem para a inovação em turismo, considerada o maior desafio competitivo atual e futuro.

\section{Proposição}

Um Sistema Regional de Inovação em Turismo representa um conjunto de competências endógenas e de competências exógenas reunidas em determinado limite geográfico e que, quando combinadas positivamente, formam um ambiente propício à geração e proposição de novas ideias e de sustentação de estratégias inovadoras de seus destinos turísticos (WEIDENFELD; WILLIAMS; BUTLER, 2010).

Sendo o turismo um setor econômico importante para a economia, conforme preconiza o T\&TCI, e a inovação o maior desafio competitivo para todos os setores, é necessário incorporar os critérios de inovação aos determinantes da competitividade em turismo e observar os condicionantes da inovação, principalmente no âmbito dos países e regiões (TRIBE, 1997).

Em atenção aos objetivos deste trabalho, a proposta do Ristur foi pensada para ser aplicada em uma escala regional de T\&T, entendendo por região um determinado aglomerado de localidades ou municípios que reúnem entre si elementos comuns em suas características geográficas, fisicas, climáticas, históricas e/ou outro fator aglutinador relevante. Portanto, os dados e fórmula de cálculo dos indicadores serão adaptados a essa escala.

O Quadro 3 apresenta a síntese da combinação entre os atributos de cada modelo - de competitividade e de inovação - e sugestão exploratória do Sistema de Inovação Regional em Turismo (Regional Innovation System in Tourism - Ristur).

Quadro 3 - Síntese do cotejamento entre pilares dos constructos

\begin{tabular}{|c|c|c|}
\hline T\&TCI & IUS & Ristur \\
\hline Ambiente habilitado & Habilitadores & Condicionantes \\
\hline 1. Ambiente de negócios & 1. Recursos humanos & 1. Capital humano em T\&T \\
\hline 2. Segurança e da segurança & $\begin{array}{l}\text { 2. Sistema P\&D atrativo, } \\
\text { aberto e excelente }\end{array}$ & 2. Sistema P\&D em T\&T \\
\hline 3. Saúde e higiene & $\begin{array}{l}\text { 3. Financiamento e suporte } \\
\text { à pesquisa }\end{array}$ & 3. Recursos naturais \\
\hline $\begin{array}{l}\text { 4. Recursos humanos/ } \\
\text { mercado de trabalho }\end{array}$ & & 4. Recursos culturais \\
\hline 5. Preparação de TIC & & 5. Ambiente global destino \\
\hline Política de T \& T & Atividades empresariais & \\
\hline $\begin{array}{l}\text { 6. Priorização de viagens e } \\
\text { turismo }\end{array}$ & 4. Investimentos em P\&D & Determinantes \\
\hline 7. Abertura international & $\begin{array}{l}\text { 5. Empreendedorismo e } \\
\text { cooperação }\end{array}$ & $\begin{array}{l}\text { 1. Empreendedorismo e } \\
\text { cooperação em T\&T }\end{array}$ \\
\hline 8. Competitividade em preço & 6. Capital intelectual & $\begin{array}{l}\text { 2. Priorização de viagens e } \\
\text { turismo }\end{array}$ \\
\hline $\begin{array}{l}\text { 9. Sustentabilidade } \\
\text { ambiental }\end{array}$ & & 3. Diferenciais competitivos \\
\hline
\end{tabular}


Quadro 3 - Continuação

\begin{tabular}{|l|l|l|}
\hline T\&TCI & IUS & Ristur \\
\hline Infraestrutura & Resultados & 4. Atrativos inovadores \\
\hline $\begin{array}{l}\text { 10. Infraestrutura de } \\
\text { transporte aéreo }\end{array}$ & 7. Inovadores & 5. Serviços turísticos inovadores \\
\hline $\begin{array}{l}\text { 11. Infraestrutura portuária } \\
\text { 12. Serviços turísticos }\end{array}$ & 8. Impactos econômicos & \\
\hline $\begin{array}{l}\text { Recursos naturais e } \\
\text { culturais }\end{array}$ & & Transformadores \\
\hline $\begin{array}{l}\text { 13. Recursos naturais } \\
\text { 14. Recursos culturais }\end{array}$ & & 2. Prosperidade social \\
\hline & Fonte - Elaborado pela autora \\
\hline
\end{tabular}

Assim como propõe o IUS (UNU-MERIT, 2011), o Ristur também supõe uma sequência causal entre as variáveis, expressas nas denominações das três diferentes dimensões de análise, e dispostas de forma que insinuem um processo de causa e consequência. 0 que significa que a inovação em turismo pode ser estimulada a partir da capacitação regional para alcançar as condições básicas favoráveis à inovação.

A análise prospectiva das condições competitivas e oportunidades de inovação em destinos turísticos pelo cruzamento das informações resultará na classificação das regiões estudadas, inspiradas nas categorias propostas, como segue:

Região Turística Líder (RTL): reúne estrategicamente os critérios que condicionam a inovação. Apresenta a melhor combinação de fatores indutores da inovação. Regiões líderes em segmentos turísticos e que exploram suas competências endógenas de forma estratégica e articulada; apresentam vantagens competitivas genuínas que, quando inspiradas nos condicionantes da economia da experiência, podem gerar oportunidades de inovação de valor em turismo e sustentar competitividade em longo prazo.

Região Turística Seguidora (RTS): reúne parte dos critérios que condicionam a inovação e apresenta boa combinação de fatores que a induzem. Regiões turísticas importantes, mas que não ocupam posição de liderança em seus segmentos e não exploram adequadamente/criativamente os recursos endógenos. Podem apresentar potencial para explorar melhor suas competências. Apresentam vantagem competitiva diferencial na oferta e experiência turística que podem inspirar importantes diferenciais.

Região Turística Modesta (RTM): não atende apropriadamente aos critérios que condicionam a inovação, ou porque não reúne recursos endógenos capazes de gerar diferenciais, ou porque não usa da criatividade para transformar seus recursos em atrativos. Não desenvolve estratégias competitivas baseadas em suas competências e a oferta não apresenta diferenciais competitivos significativos. Baseia-se na imitação e reprodução, sem originalidade na experiência turística. Participação tímida em termos de competitividade de destinos.

Essa classificação possibilitará a sistematização de tipologias de regiões de acordo com a capacidade de reunir os atributos considerados fundamentais no estímulo à inovação e à habilidade para transformar essa capacidade em estratégia competitiva inovadora em turismo. 


\section{CONSIDERAÇõES FINAIS E CONTINUIDADE DA PESQUISA}

Tendo em vista os objetivos e alcance específico deste trabalho, não cabem aqui conclusões, mas sim algumas considerações sobre sua continuidade, uma vez que se trata de parte de um projeto mais amplo e de pesquisa permanente. No entanto, essa etapa exploratória e inicial da pesquisa se mostrou fundamental para o entendimento da evolução do conhecimento sobre os temas de competitividade e de inovação, principalmente sob a perspectiva do turismo e da gestão de destinos turísticos.

A primeira parte tratou de confrontar o constructo escolhido para definir e avaliar inovação em países, com o constructo que sistematiza e avalia competitividade dos países em turismo. Essa análise foi feita através do confronto entre as macrodimensões e pilares dos modelos e demonstrou convergência direta entre parte dos indicadores e relação indireta ou complementar entre outra parte dos indicadores.

Constatou-se que o IUS contempla em seus indicadores elementos de competitividade que afetam diretamente o turismo, como é o caso da formação e fomento para a pesquisa, ciência e tecnologia, empreendedorismo, cooperação, ambiente empresarial favorável, negócios inovadores, ao mesmo tempo que o T\&TCI aborda elementos determinantes da capacidade de inovação dessas regiões, quando se preocupa com os recursos locais endógenos, a sustentabilidade socioambiental, a qualificação para o trabalho, a formação profissional e a cooperação intersetores como importantes influenciadores da capacidade competitiva para os países e regiões específicas.

Além do mérito pela objetivação de conceitos complexos como os de competitividade e inovação, esses constructos, uma vez que assumem formato de metodologia de avaliação desses fenômenos no âmbito dos países, também colocam em destaque a necessidade do monitoramento permanente de fatores críticos relativos ao desempenho competitivo de setores e países por um lado, e por outro, o de avaliar a capacidade de inovação também de países e regiões como um desses fatores críticos.

A premissa básica da pesquisa é que a inovação é o principal desafio competitivo para destinos turísticos na atualidade e continuará sendo no futuro. Portanto, a tese principal é que os dois temas devem ser analisados sob um mesmo constructo. 0 cotejamento realizado na primeira etapa permitiu a sistematização da proposição exploratória de um modelo conceitual do que pode representar um sistema de inovação em regiões turísticas, aqui denominado Ristur.

A proposta foi estruturada em três grandes dimensões: condicionantes, determinantes e transformadores. Cada dimensão contém entre três e cinco variáveis que serão definidas por meio de um conjunto de indicadores, na tentativa de construir um modelo estrutural para avaliar a capacidade de inovar em turismo, e classificar essas regiões em uma tipologia com três categorias básicas. A próxima e importante etapa da pesquisa se ocupará de validar o modelo conceitual, para então concentrar esforços em atribuir valor aos indicadores e definir objetivamente seu conteúdo.

O Ristur está proposto como um modelo sistêmico e sugere uma clara relação causa-efeito entre diferentes categorias de fatores (enablers) que influenciam a inovação e a competitivdade de regiões turísticas em longo prazo (outputs). Isso significa que o entendimento nesta pesquisa é de que a inovação pode e deve ser 
estimulada pelo aperfeiçoamento dos indicadores, que são condicionantes e fundamentais para iniciar um processo virtuoso para posicionamentos competitivos mais favoráveis e "azuis". A proposta também procura destacar a relevância da distinção (e gap) entre recursos endógenos originais e conjunto de atrativos (set resources $x$ core atractions) para as decisões estratégicas, o que significa que o importante é o que se faz com o que se tem, e não o que se tem e não se faz.

Por fim, a continuidade da pesquisa se dedicará ao teste e aperfeiçoamento do modelo para que sirva de base metodológica para estudos sobre inovação em turismo com foco na competitividade de destinos. Seus resultados poderão ainda ser aplicados ao planejamento e à gestão de regiões turísticas, e orientar a definição de estratégias regionais inovadoras, uma vez que pode servir para avaliar as condições e potencial para inovação regional, indicar oportunidades a partir de suas próprias competências, estimular o empreendedorismo, subsidiar o desenvolvimento de produtos e serviços inovadores e contribuir efetivamente para melhorar a competitividade de destinos turísticos.

\section{REFERÊNCIAS}

BRASIL. Ministério do Turismo; FGV - FUNDAÇÃO GETULIO VARGAS; SEBRAE - SERVIÇO BRASILEIRO DE APOIO ÀS MICRO E PEQUENAS EMPRESAS. Estudo de Competitividade dos 65 Destinos Indutores do Desenvolvimento Turístico Regional: Relatório Brasil. Brasília, DF: Ministério do Turismo, 2008.

BRYMAN, A. Social Research Methods. Oxford: Oxford University Press, 2004.

BUHALIS, D. The tourism phenomenon: the new tourist and consumer. In: WAHAB, C.; COOPER, C. (Eds.) Tourism in the age of globalization. London: Routledge, 2000. p. 69-96.

CROTTI, R.; MISRAHI, T. (Eds.). The travel \& tourism competitiveness report 2015: growth thought shocks. Geneva: World Economic Forum, 2015. Disponível em: <http://bit. ly/1KhKogv>. Acesso em: 4 mar. 2015.

DOEL, M.; HUBBARD, P. Taking world cities literally: Marketing the city in a global space of flows. City: analysis of urban trends, culture, theory, policy, action, [s. l.], v. 6, n. 3, p. 351-358, 2002.

DWYER, L.; KIM, C. Destination competitiveness: determinants and indicators. Current Issues in Tourism, London, v. 6, n. 5, p. 369-414, 2003.

GENEST, J.; LEGG, D. Premier-ranked tourist destinations: development of a framework for analysis and its self-guided workbook. Ontario, 2003. Disponível em: <http://www.tourism. gov.on.ca/english/research/pdf/self-guided-workbook.pdf.> Acesso em: 20 abr. 2004.

GOOROOCHURN, N.; SUGIYARTO, G. Competitiveness indicators in the travel and tourism industry. Tourism Economics, London, v. 11, n. 1, p. 25-43, 2005. Disponível em: <http:// bit.ly/2p1I60W>. Acesso em: 5 abr. 2017.

HALL, M.; WILLIAMS, A. Tourism and Innovation. London: Routledge, 2008.

HJALAGER, A. A review of innovation research in tourism. Tourism Management, London, v. 31, n. 1, p. 1-12, 2010.

HORNER, S.; SWARBROOKE, J. O comportamento do consumidor no turismo. São Paulo: Aleph, 2002.

KIM, W.; MAUBORGNE, R. A estratégia do oceano azul. Rio de Janeiro: Elsevier, 2003. 
LIPMAN, G.; VORSTER, S. Green growth, travelism, and the pursuit of happiness. In: BLANKE, J.; CHIESA, T. (Eds.). The travel \& tourism competitiveness report 2011: beyond the downturn. Geneva: World Economic Forum, 2011. p. 77-80. Disponível em: <http:// www3.weforum.org/docs/WEF_TravelTourismCompetitiveness_Report_2011.pdf $>$. Acesso em: 4 mar. 2012.

LONGHI, C. Local systems and the network in the globalisation process. In: CURZIO, A. Q.; LOPES, M. Research, technology and innovation. Heildeberg: Physica-Velag, 2005. p. 81-108.

LOPEZ, M. Innovation, competitiveness and development: searching for the linkages to economic development. Aalborg: Aalborg University, 2000.

LUNDVALL, A. et al. National systems of production, innovation and competence building. Research Policy, Amsterdam, v. 3, n. 2, p. 213-231, 2002.

MARTON-LEFÈVRE, J.; BORGES, M. A new big plan for nature: opportunities for travel \& tourism. BLANKE, J.; CHIESA, T. (Eds.). The travel \& tourism competitiveness report 2011: beyond the downturn. Geneva: World Economic Forum, 2011. p. 81-88. Disponível em: <http://www3.weforum.org/docs/WEF_TravelTourismCompetitiveness_Report_2011. pdf>. Acesso em: 7 jun. 2012.

MAZARO, R. M. Atualização da sustentabilidade estratégica como instrumento de gestão de destinos turísticos. Revista Turismo \& Desenvolvimento, Campinas, n. 13/14, p. 771781, 2010.

OECD - ORGANIZATION FOR ECONOMIC COOPERATION AND DEVELOPMENT. Innovation and knowledge-intensive service activities. 3. ed. Paris, 2006.

. Conference on innovation and growth in tourism. Lugano, 2003.

PINE, B.; GILMORE, J. The experience economy: work is theatre and every business a stag. Boston: Harvard Business School Press, 1999.

RITCHIE, J.; CROUCH, G. The competitive destination: a sustainable tourism perspective. Wallingford: CAB International, 2003.

RODRÍGUEZ, I.; WILLIAMS, A.; HALL, M. Tourism innovation policy: implementation and outcomes. Annals of Tourism Research, Amsterdam, n. 49, p. 76-93, 2014.

SARKAR, S. Empreendedorismo e inovação. Lisboa: Escolar, 2010.

SIPE, L.; TESTA, M. What is innovation in the hospitality and tourism marketplace? A suggested research framework and outputs typology, hospitality \& tourism management. In: INTERNATIONAL CHRIE CONFERENCE-REFEREED TRACK, 22., 2009, Amherst. Anais... Amherst: University of Massachusetts, 2009.

SOETE, L. The costs of a non-innovative Europe. 2010. Disponível em: <http://www.eurosfaire.prd.fr/7pc/doc/1286524609_demeter_costs_non_innovative_europe_unu_merit.pdf>. Acesso em: 30 mar. 2012.

TRIBE, J. The indiscipline of tourism. Annals of Tourism Research, Amsterdam, v. 24, n. 3, p. 638, 1997.

. Strategy for Tourism. 2. ed. Oxford: Goodfellow, 2015.

TROUT, J. Diferenciar ou morrer: sobrevivendo em uma era de competição mortal. São Paulo: Futura, 2000.

UNU-MERIT. Innovation Union Scoreboard: the Innovation Union's performance scoreboard for research and innovation, 2011. Disponível em: <http://bit.ly/2oDRn2z>. Acesso em: 13 jul. 2012.

. Innovation Union Scoreboard 2015: the Innovation Union's performance scoreboard, 2015. Disponível em: <http://bit.ly/2oJoJKJ>. Acesso em: 13 mar. 2016. 
WEF - WORLD ECONOMIC FORUM. The travel \& tourism competitiveness report 2007: furthering the process of economic development. 2007. Disponível em: <http://bit. ly/2nbUUoL $>$. Acesso em: 9 mar. 2008.

The travel \& tourism competitiveness report 2011: beyond and downturn. 2011. Disponível em: <http://bit.ly/2oaPmua>. Acesso em: 4 mar. 2012.

. The travel \& tourism competitiveness report 2013: reducing barriers to economic growth and job creation. Disponível em: <http://bit.ly/1i4SD1I >. Acesso em: 10 mar. 2014.

. The travel \& tourism competitiveness report 2015: growth thought shocks. Disponível em: <http://bit.ly/1EgtXgK>. Acesso em: 4 mar. 2015.

WEIDENFELD, A.; WILLIAMS, A.; BUTLER, R. Knowledge transfer and innovation among attractions, Annals of Tourism Research, Amsterdam, v. 37, n. 3, p. 604-626, 2010.

WTO - WORLD TOURISM ORGANIZATION. Tourism 2020 vision: global forecasts and profiles of market segments. Madrid, 2006.

XIN, S.; TRIBE, J.; CHAMBERS, D. Conceptual Research in Tourism, Annals of Tourism Research, Amsterdam, v. 41, p. 66-88, 2013.

Recebido em: 03/12/2015

Aprovado em: 17/11/2016 